It may be of interest to record that on the Gardens side the principle of exchange has for some time been in active operation, as we now exchange student gardeners with Germany, France, Italy, Sweden, Switzerland, Belgium, Canada, the United States of America and New Zealand, and other parts of the Empire when opportunity offers. The Kew student is sent out in exchange for one or two years, his place being taken by a student gardener from the botanic garden or other centre to which he has been sent. At the present time, twelve exchanges are in operation, much to the advantage of the men coneorned and certainly to the advantage of Kew.

\title{
Wetting and Detergent Action
}

$\mathrm{T}$ HE symposium on the "Scientific and Technical Aspects of Wetting and Detergency", held at the Imperial College of Science on February 19-20 under the auspices of the British Section of the International Society of Leather Trades Chemists, brought together a large company of scientific and technical workers for the discussion of nearly twenty interesting papers, which dealt with many of the known important points in these two problems, and suggested new points of view likely to prove important in future research.

The fundamental quantities determining whether a solid is, or is not, completely wetted by a liquid, are the surface tensions of the solid, and the liquid, and the amount of adhesion between the solid and the liquid. The impossibility, at present, of measuring the surface tension of solids is not such a serious difficulty in the scientific study of wetting as might be supposed, since the contact angle, which can be measured, is simply related to the surface tension of the liquid and the adhesion between solid and liquid, and is itself the immediately relevant quantity in most problems of wetting; and probably also in detergency.

C. G. Sumner described modifications in the 'plate' method for determining contact angles ; N. K. Adam showed how contact angles can be determined on single textile fibres or thin wires visible under low powers of the microscope; and J. O. Cutter and C. W. Price reported a careful study of the method (originally due to Bartell and Osterhof) of measuring the adhesion tension and contact angles of liquids against solid powders, by determination of the pressure required to prevent the liquid penetrating into solid plugs of the powder, kept compact under high compression. This method, the only one available for the quantitative study of the wetting of fine powders, is not easy ; it depends on careful attention to details of the apparatus and on the maintenance of a high pressure on the powder.

Probably the most important single industry dependent on the wetting properties of solid surfaces is that of mineral flotation; H. Freundlich dealt, in a short paper with a valuable bibliography, with the principal features of this process, including a discussion of the importance of contact angle, and of the surface films formed by the selective adsorption of the 'collectors' on the surface of the valuable minerals, which decrease their ease of wetting by the water and consequently cause them to stick to the air bubbles rising in the froth, and thus become collected at the surface. H. Martin described the very important applications of wetting agents in assisting the spread of insecticide solutions over the surface of leaves and fruit. $\mathrm{He}$ has taken particular account of the difference between the advancing and the receding contact angles, directing attention to which of the two is relevant for each practical problem; and he deserves gratitude for his timely protest against the pernicious and short-sighted practice of some manufacturers, in placing on the market, and expecting intelligent and chemically minded customers to use, or advisory officers to recommend, mixtures of unspecified and possibly inconstant composition bearing names suggestive of 'nostrums' rather than of scientifically manufactured and reliable products.

A paper by E. J. Daniels and D. J. Macnaughtan directed attention to some effects of surface impurities and inclusions in metals, on their ease of wetting by solders or by tin; this is a very important subject to which but little quantitative study appears to have been given. A. de Waele discussed the importance of wetting in the deflocculation and the plasticity of solid suspensions; and W. E. Wornum and G. A. Campbell dealt with the wetting problems of the paint industry, including the fundamental problem of the wetting of pigments by the liquid medium, and that of the surfaces to be painted by the finished paint or varnish. Wetting, it must be remembered, is not identical with deflocculation or dispersion; it is but one of the two main factors concerned. The other is the adhesion of the solid particles for each other, which must be overcome by the wetting liquid for the dispersion to be effective.

Detergency, which formed the subject of the second day's discussion, is a more complicated and perhaps more interesting problem. C. Robinson, in his introductory paper, discussed a theory due to N. K. Adam which places the emphasis in detergent action on the displacement of the grease from the solid surface, as comparatively large globules; the contact angle formed by the surface of separation between grease and aqueous detergent solutions, and the solid being cleaned, being the important measurable quantity determining the efficiency of detergent action. If this is correct, detergent action is essentially a preferential wetting of the solid surface by the detergent, instead of by the grease, and not, as has been occasionally held in the past, simply a wetting of the grease by the detergent, with consequent emulsification of the grease or other dirt. Surface and interfacial tensions, of course, enter into the problem, but neither alone is necessarily of predominating importance. Robinson considered also the problem of the removal of detached globules of grease from entanglement with the eloth; and described his measurements of the interfacial tension between oils and detergent solutions, which show some very remarkable effects of traces of polyvalent salts. G. S. Hartley discussed the power that solutions of soaps and paraffin chain colloidal nlectrolytes ('soap-like' substances) possess, of actually dissolving organic compounds which are 
insoluble in water. These substances, even in quite dilute solutions, have their.long paraffin chain ions aggregated into 'ionic micelles', and Hartley suggests that the solvent power is due to the interior of these micelles closely resembling liquid paraffin, and having similar solvent properties; so that when such a substance as azobenzene, or cetyl alcohol, is caused to dissolve in a soap solution, it is not distributed evenly over the whole solution, but is all to be found in the interior of the ionic micelles. A discussion of the changes in vapour pressure, as the ionic micelles increase in size through taking up other organic substances, leads to the conclusion that they can increase only to a certain size before becoming unstable; hence the solvent power of a soap solution for organic substances insoluble in water is definite and limited, and there is an unstable region of sizes between the largest micelles which can exist, containing dissolved organic material, and the droplets of an emulsion stabilized by a monolayer of the soaplike material : these emulsions simulate stability, though they are not strictly stable in a thermodynamic sense. Whether such solvent action has any practical bearing on the problem of detergent action is not certain; but it is a most interesting and novel theory of solvent action in unusual circumstances. E. K. Rideal directed attention to the possibility that the molecules of detergent substances may penetrate and displace films of other substances adhering to a solid surface; that such penetration often occurs in monolayers at an air-water surface has been shown by J. H. Schulman and A. H. Hughes. An extension of v. Búzagh's work on the adhesion of solid particles to solid faces was also described, with particular reference to detergent solutions.

Space scarcely permits of an adequate notice of the important points in the chemical constitution of detergent and wetting agents, but papers by $\mathrm{H}$. $\mathrm{K}$. Dean, and by E. T. Williams, C. B. Brown and H. B. Oakley gave a general account of them. As a rule, the successful detergents have a long hydrocarbon chain attached to an ionizable, strongly acidic, end group ; compounds of similar constitution but basic, yielding paraffin chain cations, may be good detergents but suffer from the practical difficulty that, being mutually precipitated by soaps, they cannot be utilized in conjunction with soaps. One case was mentioned, however, of a new detergent in which there. is no jonized end group, but instead a polymerized glycerol derivative, partially esterified with fatty acids.

That wetting and detergent agents operate by forming surface films on the surface both of the solid and of the grease or other dirt to be removed is clear. Part of the discussion turned on the orientation of the molecules in these films. Though the proof is not complete, it appears probable that when the wetting of a greasy surface, that is, one externally composed mainly of paraffin groups, is assisted by a wetting agent, the hydrocarbon part of the molecule of the wetting agent lies flat on the surface; the observed increase in hydrophilic character can probably be accounted for by the presence of only one water-attracting group in the space on the surface occupied by a molecule of the wetting agent lying flat. Such observations as are available on the amount of adsorption of detergents by paraffin wax surfaces show it to be much less than that required to cover the surface with a monolayer oriented perpendicular to the surface, but of the right order for a complete layer of molecules lying flat. In cases where a soluble agent is required to diminish the ease of wetting of a solid surface, as with the collectors used in flotation, the molecules may be oriented perpendicular to the surface, but with the hydrophilic groups towards the solid; this is not yet, however, certain, as a considerable diminution of wetting could probably be attained even with the molecules lying flat. A rather interesting case of the deposition of a layer difficult to wet on glass was mentioned; substances such as cetyl trimethyl ammonium salts, with a long paraffin chain cation, cause (in dilute solution) clean glass to become greasy in appearance, probably through their hydrophilic ends being attracted to the glass, which thus acquires a paraffinlike exterior.

The papers presented will be published in a separate volume, shortly; and they constitute $a_{0}$ valuable focusing of ideas on many very importent, and not yet fully understood, industrial questions.

\section{N. K. A.}

\section{Maiden Castle, Dorchester}

\begin{abstract}
A REPORT by Dr. Mortimer R. E. Wheeler on the results of three years' work on the hill-top site of Maiden Castle, Dorchester, on behalf of the Society of Antiquaries and the Dorset Archæological Society, was presented at a meeting of the former Society on February 25.

As originally planned, the excavation was intended to occupy three seasons. The third and last of these in the autumn of 1936 was prolonged until December 24. As, however, the site has proved of even more intense interest than was anticipated, providing evidence of signal importance in its bearing on the development of civilization in the south and southwest of Great Britain, it has been decided to extend the investigation for another season to enable supplementary excavations to be carried out next year.

Maiden Castle has always been impressive by its size alone; and its vast dimensions have borne witness to a capacity for economic and political
\end{abstract}

organization, which it seemed difficult to credit to a population so primitive as the early inhabitants of Britain. Dr. Wheeler's excavations have shown that not only was the construction of a far more complex and imposing character than surface indication showed, but also that it embodies a complete picture of all known phases of urban life in Britain before the days of the building of Roman towns in the latter part of the first century; while the presumedly primitive character of early British culture is shown to be anything but in accord with the facts now revealed.

Dr. Wheeler distinguishes at least four major phases in the occupation of Maiden Castle. The first, belonging to the early neolithic, was a settlement at the eastern end of the mound which had long been covered over, and became known only by this excavation. It had been enclosed by at least three rings of entrenchment excavated in the chalk by deer-horn picks. A large section of this was uncovered 